\title{
Characterizing Fabric Anisotropy of Air-Pluviated Sands
}

\author{
Quan $\mathrm{Sun}^{1}$, Junxing Zheng ${ }^{1, *}$, Hantao $\mathrm{He}^{1}$, and Zhaochao $\mathrm{Li}^{1}$ \\ ${ }^{1}$ Iowa State University, Department of Civil, Construction and Environmental Engineering, Ames, USA
}

\begin{abstract}
Sand particles depositing through air generally align their largest dimensions in horizontal plane, forming a cross anisotropic fabric. Therefore, sands display varying strength, permeability, compressibility with directions. This study characterizes fabric anisotropy in loose and dense air-pluviated sand specimens scanned by X-ray Computed Tomography (X-ray CT) using a series of image processing techniques. The principal component analysis, three-dimensional watershed analysis, and Delaunay triangulation technique are used to compute directional parameters, including particle long axes, contact normals, and branch vectors, and scalar parameters, including index void ratios, coordination number, and average branch vector length. The particle long axes and branch vectors displayed preferred horizontal directions while the contact normals displayed preferred vertical directions. The dense specimen has smaller index void ratios, larger coordination number, and smaller average branch vector length than the loose specimen.
\end{abstract}

\section{Introduction}

In granular soils, particles depositing through air and water generally align their largest dimensions in horizontal plane, forming a cross anisotropic fabric. The importance of fabric anisotropy on the mechanical behavior of sands, such as strength, permeability, and compressibility, was demonstrated by numerous experimental studies and discrete element method (DEM) studies. Some typical works included Tatsuoka [1], Azami et al. [2] , Lade [3], Yang et al. [4], Hosseininia [5], Fonseca et al.[6, 7], Zhao and Guo [8, 9], Imseeh et al. [10], and Mahbub and Haque [11].

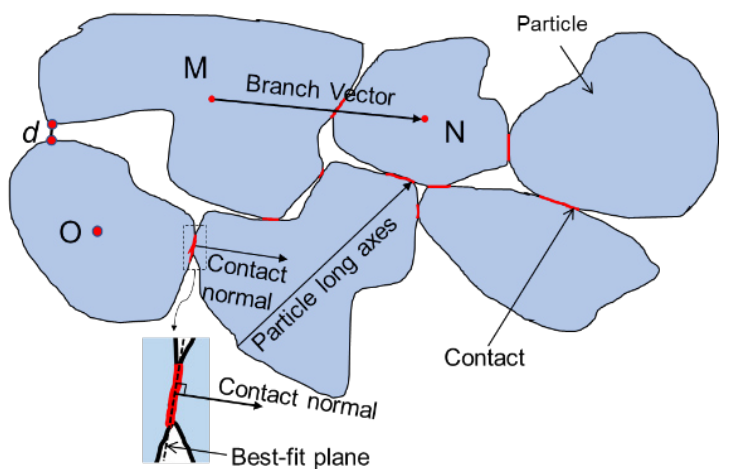

Fig. 1. Schematic diagram of directional parameters for fabric characterization.

Soil fabric can be described by spatial orientations of directional parameters, such as particle long axes, contact normals, and branch vectors as shown in Fig. 1. Soil fabric can also be quantified by scalar parameters, such as index void ratios, coordination number, and average branch vector length.

Two-dimensional (2D) image processing techniques (Yang et al. [12] and Zheng and Hryciw [13, 14]) have been developed to compute directional and scalar parameters and characterize fabric anisotropy from 2D images. However, these methods cannot characterize fabric anisotropy in three-dimensional (3D) soil specimen. A number of studies such as Fonseca et al. $[6,7]$, Alam et al. [15], Imseeh et al. [10], and Mahbub and Haque [11] used X-ray CT to investigate effects of 3D fabric on strength and compressibility of sands in triaxial tests and oedometer tests. However, to date, there was a lack of studies characterizing fabric anisotropy in air-pluviated sand specimen in loose and dense conditions. This study aimed to fill this gap.

A river sand specimen (containing sub-angular to well-rounded particles) was prepared at loose and dense conditions. Both loose and dense specimens were scanned by X-ray CT to generate volumetric images. A series of computational geometry techniques were developed to analyze volumetric images to determine directional and scalar parameters. The $3 \mathrm{D}$ rose diagram and density function were used to exhibit the spatial distribution of directional parameters. In addition to granular soils, the developed methodologies can be applied to characterize fabric anisotropy of other

\footnotetext{
${ }^{*}$ Corresponding author: junxing@iastate.edu
} 
particulate materials, such as ore, powders, chemicals, pharmaceuticals, and food products.

\section{Fabric Characterization}

The fabric in a granular soil can be quantified by directional and scalar parameters following Fonseca et al. $[6,7]$. The directional parameters include particle long axes, contact normal, and branch vectors. The scalar parameters include void ratio, coordination number, and average branch vector length. Spatial distributions of particle long axes, contact normals, and branch vectors in $3 \mathrm{D}$ space are usually plotted as 3D rose diagrams. For example, this paper generates a random distribution of vectors in $3 \mathrm{D}$ space. These vectors are plotted as a 3D rose diagram in Fig. 2(a). Each bar represents frequency of vectors in this direction in $3 \mathrm{D}$ space.

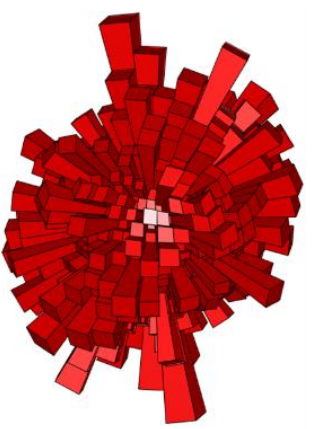

(a) Rose diagram

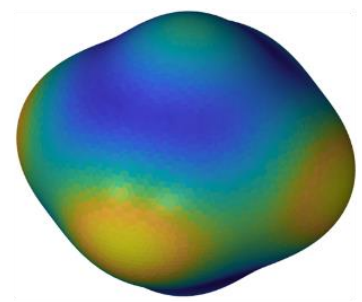

(b) Fitting surface by $f(n)$
Fig. 2. The rose diagram and density function $f(n)$.

The rose diagram can be represented by a density function $f(n)$ proposed by Kanatani [16]:

$$
f(n)=\frac{1}{2 \pi}\left[1+D_{i j}^{*} n_{i}^{*} n_{j}^{*}+D_{i j k l}^{*} n_{i}^{*} n_{j}^{*} n_{k}^{*} n_{l}^{*}\right],
$$

Where the superscript * represents either ' $p$ ', 'c' or ' $b$ ' meaning particle long axes, contact normals or branch vectors; $D_{i j}^{*}$ is the second order deviatoric fabric tensor; and $D_{i j k l}^{*}$ is the fourth order deviatoric fabric tensor:

$$
\begin{gathered}
D_{i j}^{*}=\frac{2}{15}\left(\varphi_{i j}^{*}-\frac{1}{3} \delta_{i j}\right), \\
D_{i j k l}^{*}=\frac{2}{15}\left(\varphi_{i j k l}^{*}-\frac{6}{7} \delta_{(i j} \varphi_{k l)}^{*}+\frac{3}{35} \delta_{(i j} \delta_{k l)}\right),
\end{gathered}
$$

In Eqs. (2) and (3), the $\delta_{i j}$ is the Kronecker delta and the $\varphi_{i j}^{*}$ and $\varphi_{i j k l}^{*}$ are second order and fourth order fabric tensors respectively:

$$
\begin{aligned}
\varphi_{i j}^{*} & =\frac{1}{N^{*}} \sum_{N_{*}} n_{i}^{*} n_{j}^{*}, \\
\varphi_{i j k l}^{*} & =\frac{1}{N^{*}} \sum_{N_{*}} n_{i}^{*} n_{j}^{*} n_{k}^{*} n_{l}^{*},
\end{aligned}
$$

Where $N^{*}$ is the total number of vectors $n^{*}(*=\mathrm{p}, \mathrm{c}$, b) in the assembly of particles.

The vectors Fig. 2(a) are used to compute the density function by using Eqs. (1) to (5). The result is shown in Fig. 2(b). The density function is essentially the bestfitting surface of the 3D rose diagram.

For a 3D soil specimen, particle long axes, contacts and contact normals, and branch vectors can be computed using a series of computational geometry techniques developed in this study. These directional parameters can be plotted in rose diagram and can be used to compute density functions using Eqs. (1) to (5). The $3 \mathrm{D}$ rose diagram and density function are used as measures in this study to characterize fabric anisotropy.

\section{Three-dimensional Volumetric Soil Specimen Scanned by X-ray CT}

A natural river sand was used in this study to develop the methodology. The river sand contained particles from sub-angular to well-rounded. A total of 2925 particles in the size range from $\# 30$ sieve $(0.595 \mathrm{~mm})$ to \#16 sieve $(1.19 \mathrm{~mm})$ were selected. These particles were carefully funnelled into a $12 \mathrm{~mm}$ diameter plastic cylinder to create a loose specimen with a height of $21.5 \mathrm{~mm}$. After scanning the loose specimen by X-ray $\mathrm{CT}$, the cylinder was vibrated to create a dense specimen with a height of $18.5 \mathrm{~mm}$, which was also scanned by Xray $\mathrm{CT}$ to acquire a volumetric image.

The 3D watershed analysis was performed on images of loose and dense specimens. A total of 2925 particles were obtained in both cases, which agreed with the true number of particles. The obtained individual particles are shown in Figs. 3(a) and (b) respectively.

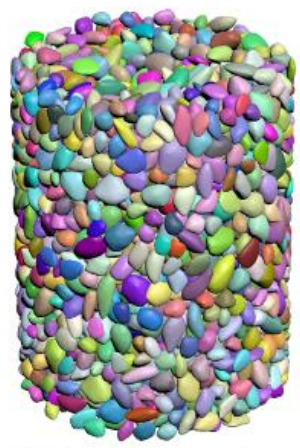

(a) The loose specimen

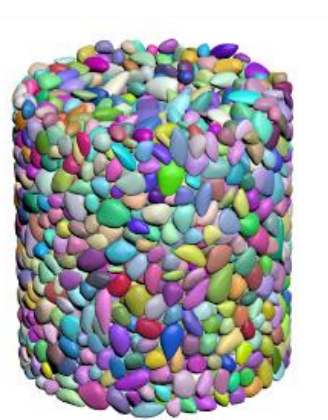

(b) The dense specimen
Fig. 3. The loose and dense sand specimen scanned by X-ray CT

\section{Characterization of Fabric Anisotropy Using Directional Parameters}

\subsection{Particle Long Axes}

The particle long axes can be determined by a principal component analysis (PCA) technique proposed by Pearson [17]. The basic concept is to project a cloud of 
points into orthogonal directions, called principal components. The first component has the largest possible variance of the data points, which is the largest dimension (long axis) of the object.

The PCA was performed on the 2925 particles in loose and dense specimens in Figs. 3(a) and (b), respectively. The determined particle long axes are shown in Figs. 4(a) and (b), respectively.

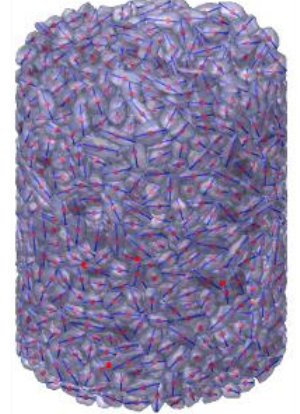

(a) The loose specimen

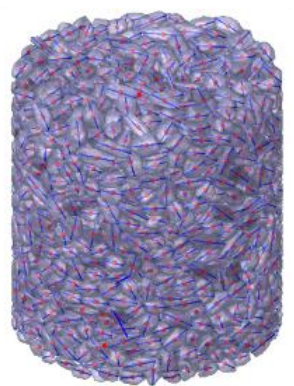

(b) The dense specimen
Fig. 4. Particle long axes of loose and dense specimens by principal component analysis.

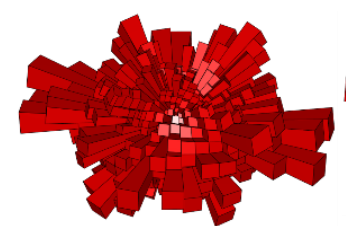

(a) The loose specimen

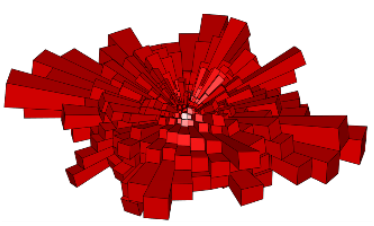

(b) The dense specimen
Fig. 5. The rose diagrams of particle long axes of loose and dense specimens.

The $3 \mathrm{D}$ rose diagrams of particle long axes in loose and dense conditions are shown in Figs. 5(a) and 5(b) respectively. The density functions $f(n)$ of loose and dense conditions are shown in Figs. 6(a) and (b) respectively. Expectedly, the particle long axes of airpluviated sand specimen displayed a preferred horizontal orientation in both loose and dense conditions. The particle long axes displayed a stronger preferred horizontal orientation in the dense condition than the loose condition.

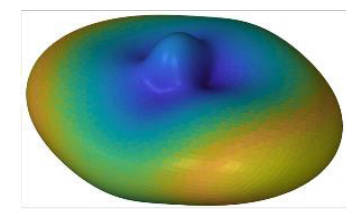

(a) The loose specimen

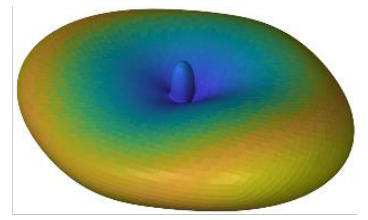

(b) The dense specimen
Fig. 6. The density functions of particle long axes of loose and dense specimens.

\subsection{Contact Normal}

The segmentation planes generated by the improved watershed analysis identify the inter-particle contacts. A total of 12357 contacts were identified in the loose specimen and 12551 contacts were identified in the dense specimen, which are plotted in Figs. 7(a) and (b) respectively. Then the points on the contacts were used to determine best-fit planes by the least square regression to determine contact normals.

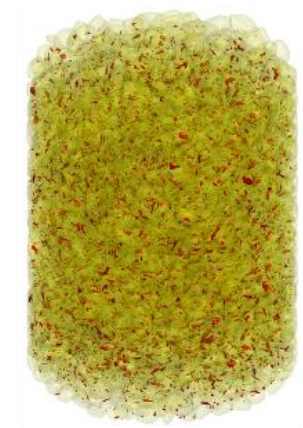

(a) The loose specimen

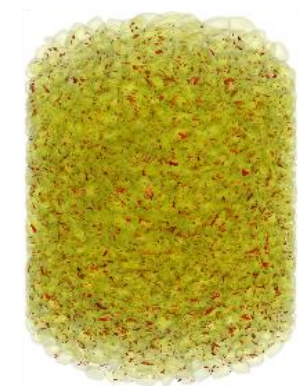

(b) The dense specimen
Fig. 7. Contacts of loose and dense specimens by watershed analysis.

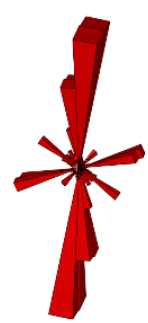

(a) The loose specimen

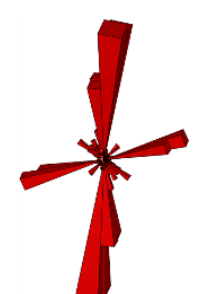

(b) The dense specimen
Fig. 8. The rose diagrams of contact normals of loose and dense specimens.

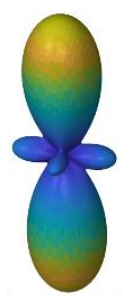

(a) The loose specimen

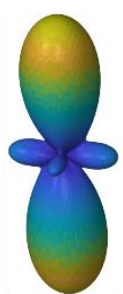

(b) The dense specimen
Fig. 9. The density functions of contact normals of loose and dense specimens.

The rose diagrams and density functions of contact normals are shown in Figs. 8 and 9 respectively. The results showed that contact normals mainly distribute in the vertical direction in air-pluviated sand specimen due to the gravity force. Therefore, the soil specimens tended to develop larger load-bearing capacity in the vertical direction than other directions. This has been confirmed by triaxial test results (Ochiai and Lade [18], Arthur and Menzies [19], Arthur and Phillips [20], and Oda et al. [21]). They observed that the mobilized angle of internal friction monotonically decreased by about $3^{\circ}$ as loading directions changed from vertical direction to horizontal direction. 


\subsection{Branch Vector}

The branch vector is the vector connecting the centroids of two contacting particles. It should be noted that the branch vector only exists between contacting particles, such as particles $\mathrm{M}$ and $\mathrm{N}$ in Fig. 1. If the particles are not contacting, such as particles $\mathrm{O}$ and $\mathrm{M}$ or particles $\mathrm{O}$ and N, no branch vectors can be constructed. This paper modified a Delaunay triangulation technique to determine branch vectors in the $3 \mathrm{D}$ volumetric images.

The Delaunay triangulation was originally proposed by Delaunay [22]. It addressed how to create an optimal triangular mesh from a set of points in 2D or 3D space by avoiding skewed and irregular triangles.

Five adjacent soil particles from the loose specimen were used to illustrate Delaunay triangulation in Fig. 10. Delaunay triangulation technique was applied to the centroids of five particles to build a 3D mesh. Three tetrahedrons, $\mathrm{ABCD}, \mathrm{ACDE}$, and $\mathrm{BCDE}$ were determined as shown in Fig. 10(b). However, the particles $\mathrm{A}$ and $\mathrm{D}, \mathrm{A}$ and $\mathrm{B}, \mathrm{A}$ and $\mathrm{E}, \mathrm{B}$ and $\mathrm{E}$, and $\mathrm{C}$ and $\mathrm{D}$ were not contacting with each other. Therefore, their centroid connections could not be used to compute the branch vectors. These connections were defined as "overconnections" and should be eliminated from the mesh. Therefore, this study modified the Delaunay triangulation to address this issue. The contacts of these five particles have been identified by watershed analysis. Therefore, this information can be used here to evaluate whether contacts exist between two connected particles and to identify the overconnections. After removing overconnections, the remaining connections were branch vectors as shown in Fig. 10(c).

The modified Delaunay triangulation technique was used to compute branch vectors in loose and dense specimens. The results are shown in Figs. 11(a) and (b) respectively. A total of 12113 branch vectors were identified in the loose specimen and 12322 branch vectors were identified in the dense specimen.

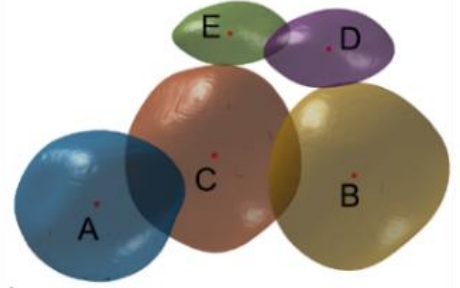

(a) 3D volumetric images of five adjacent particles.

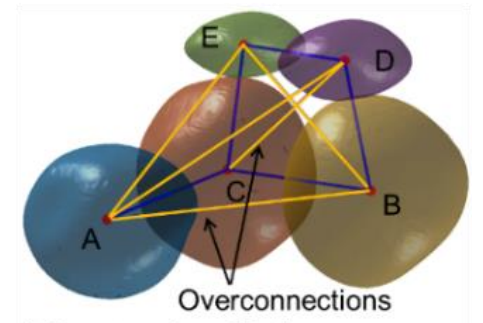

(b) Construction of Delaunay triangulation using centroids of particles.

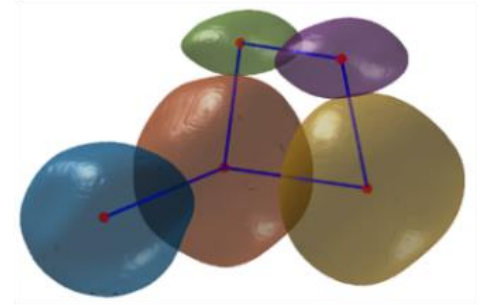

(c) The branch vectors by modified Delaunay triangulation.

Fig. 10. Illustration of modified Delaunay trianglation using five adjacent particles.

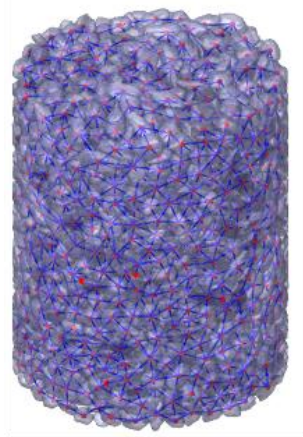

(a) The loose specimen

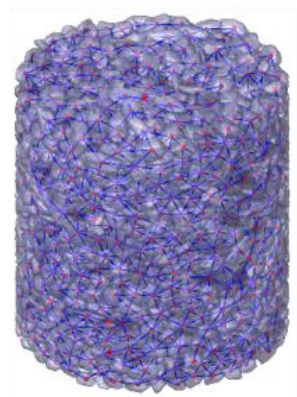

(b) The dense specimen
Fig. 11. Branch vectors of loose and dense specimens by modified Delaunay triangulation.

The rose diagrams and density functions are shown in Figs. 12 and 13. The results showed that the branch vectors displayed preferred horizontal directions. Branch vectors displayed stronger preferred horizontal direction in the dense specimen than the loose specimen. 


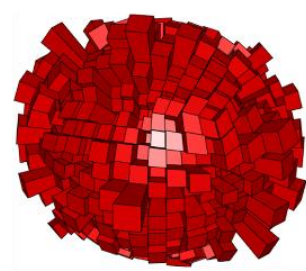

(a) The loose specimen

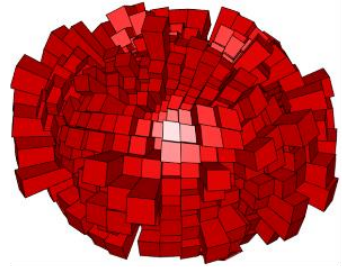

(b) The dense specimen
Fig. 12. The rose diagrams of branch vectors of loose and dense specimens.

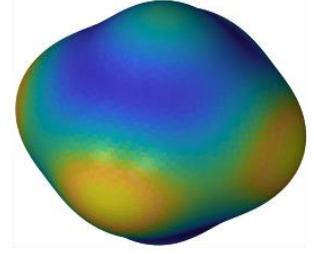

(a) The loose specimen

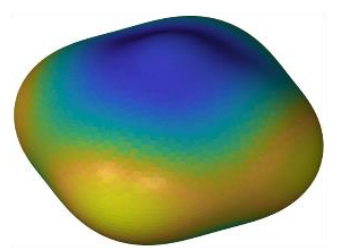

(b) The dense specimen
Fig. 13. The density functions of branch vectors of loose and dense specimens.

\section{Characterization of Fabric Anisotropy Using Scalar Parameters}

The fabric anisotropy can also be quantified by scalar parameters, including void ratios, coordination number, and average branch vector length. These parameters can be computed after identifying contacts and branch vectors.

\subsection{Void Ratios}

The void ratio can be calculated as the volume of voids over the volume of particles. In the volumetric image, the volume was calculated by the number of voxels. Therefore, following [6], the void ratio can be computed by:

$$
e_{C T}=\frac{N_{\text {total voxel }}-N_{\text {particle voxel }}}{N_{\text {particle voxel }}},
$$

Where $N_{\text {total voxel }}$ is the total number of voxels of the specimen, $N_{\text {particle voxel }}$ is the number of voxels within soil particles, and $N_{\text {total voxel }}-N_{\text {particle voxel }}$ is the number of voxels within voids. Based on Eq. (6), the void ratios of loose and dense specimens in Figs. 3(a) and (b) were calculated as 0.95 and 0.68 respectively.

\subsection{Coordination Number}

The coordination number $(\mathrm{CN})$ quantifies the average number of contacts of individual particles in a specimen:

$$
C N=\frac{2 N_{\mathrm{c}}}{N_{\mathrm{p}}},
$$

Where $N_{p}$ is the number of particles in the specimen, and $N_{c}$ is the number of contacts. The total number of contacts of the loose and dense specimens in Figs. 3(a) and (b) were determined as 12357 and 12551 respectively. The number of particles was 2925. Therefore, the CNs of loose and dense specimens were 8.45 and 8.58 , respectively. The dense specimen had a larger coordination number than the loose specimen.

\subsection{Average Length of the Branch Vector}

Since the branch vectors of the specimens were obtained previously, these results could be used to compute the length of each branch vector following [6]. The average length of the branch vectors for loose and dense specimens were $1.3 \mathrm{~mm}$ and $1.1 \mathrm{~mm}$ respectively. The dense specimen exhibited the smaller inter-particle distance than the loose specimen.

\section{Conclusion}

This paper analyzes the fabric anisotropy in airpluviated sand specimens. A river sand specimen (containing sub-angular to well-rounded particles) was prepared at loose and dense conditions. Both loose and dense specimens were scanned by X-ray CT to generate volumetric images.

Three computational geometry techniques: principal component analysis, watershed analysis, and modified Delaunay triangulation were used to analyze the volumetric images to determine key parameters for fabric characterization. The principal component analysis identified particle long axes. The watershed analysis segmented contact particles and identified contacts between particles. The modified Delaunay triangulation identified the branch vectors between contacting particles. The particle long axes, contact normals, and branch vectors were used to compute $3 \mathrm{D}$ rose diagram and density functions. The computational geometry technique could also be used to compute the scalar fabric descriptors, including index void ratios, coordination number, and the average length of branch vectors.

The particle long axes and branch vectors displayed preferred horizontal direction while the contact normals displayed preferred vertical direction. This explained that in triaxial tests, the mobilized friction angle decreases as loading direction changes from vertical to horizontal directions. The dense specimen had smaller index void ratios, larger coordination number, and smaller average branch vector length. This explained dense specimens have larger strength and dilatancy than the loose specimens. Results showed that the proposed technique successfully captured the fabric evolution between loose and dense specimens.

The authors want to acknowledge the Department of Civil, Construction and Environmental Engineering, Iowa State University for providing financial support to this research. 


\section{References}

1. F. Tatsuoka. Advanced triaxial testing of soil and rock. (1988)

2. A. Azami, S. Pietruszczak and P. Guo. Int. Jour. Fs. Num. \& Ana. M. in Geo. 34, 771 (2010)

3. P. V. Lade. Int. Jour. O. Ss. \& Ss. 44, 5146 (2007)

4. Z. Yang, X. Lit and J. Yang. Géote, (2008)

5. E. S. Hosseininia. Gran. M. 14, 483 (2012)

6. J. Fonseca, C. O'sullivan, M. Coop and P. Lee. Géote. 63, 818 (2013)

7. J. Fonseca, C. O'Sullivan, M. R. Coop and P. Lee. Géote. 63, 487 (2013)

8. J. Zhao and N. Guo. Géote. 63, 695 (2013)

9. J. Zhao and N. Guo. Géotec. 65, 642 (2015)

10. W. H. Imseeh, A. M. Druckrey and K. A. Alshibli. Gran. M. 20, 24 (2018)

11. A. Al Mahbub and A. Haque. Mats. 9, 890 (2016)

12. Z. X. Yang, X. S. Li and J. Yang. Géote. 58, 237 (2008)

13. J. Zheng and R. D. Hryciw. Coms. and Geotes. 88, 46 (2017)

14. J. Zheng and R. D. Hryciw. Ss. and Fs., (2018)

15. M. F. Alam, A. Haque and P. G. Ranjith. Mats. 11, (2018)

16. K.-I. Kanatani. Int. Jour. O. Engi. Sci. 22, 531 (1984)

17. K. Pearson. The L., E., \& D. Phil. Mag. \& Jour. of Sci. 2, 559 (1901)

18. H. Ochiai and P. V. Lade. Jour. O. Geote. Engi. 109, 1313 (1983)

19. J. R. F. Arthur and B. K. Menzies. Géote. 22, 115 (1972)

20. J. R. F. Arthur and A. B. Phillips. Géote. 25, 799 (1975)

21. M. Oda, I. Koishikawa and T. Higuchi. Ss. \& Fs. 18, 25 (1978)

22. B. Delaunay. Izv. Akad. Nauk SSSR, Ot. Mat. i Es. N. 7, 1 (1934) 